\title{
Are there speculative bubbles in stock markets? Evidence from an alternative approach*
}

\author{
Guojun Wu and ZhiJie Xiao
}

Monte Carlo evidence [Evans (1991)] indicates that when speculative bubbles are collapsible, the traditional cointegration approach based on unit root tests has some serious drawbacks. We propose in this paper an alternative approach to test such bubbles. We demonstrate that the suggested test has some advantages over the traditional unit root based tests, especially for bubbles that are collapsible. Properties of the proposed procedure are investigated. Extensions and generalizations of this procedure are also studied. The testing procedure is applied to market indexes in the U.S. and Hong Kong. The proposed procedures provide useful complements to existing bubble tests.

\section{INTRODUCTION}

There is a large collection of literature on the empirical tests of asset price behavior [see, e.g., Flood and Garber (1980), Shiller (1981), Blanchard and Watson (1982), Campbell and Shiller (1988), West (1988), Froot and Obstfeld (1991), and McQueen and Thorley (1994)]. Researchers have argued that stock prices are not consistent with the fundamentals that the prices are supposed to represent, i.e., with the discounted stream of future dividends [See, for example, Cochrane (1991)].

One possible remedy of this problem is to improve the model of fundamentals so that they more closely reflect observed asset prices. Yet, this approach has yielded limited success principally because stock prices exhibit much higher volatility than what can be explained by movements in fundamental variables such as earnings, dividends and interest rates [see, e.g., Shiller (1981)]. Moreover, dividend-based models seem to be ill-equipped to explain some of the stylized facts about stock returns. [see Cox and Ross (1976), and Bekaert and $\mathrm{Wu}(2000)]$.

An alternative approach to explaining this phenomenon is to introduce speculative bubbles into asset pricing models. Bubbles could explain the empirical features of stock returns [see, inter alia, Flood and Hodrick (1986), and Camerer (1989)]. Time-varying volatility would be a natural consequence of the presence of bubbles. Stock volatility may be high or low, as the size of the bubble grows or shrinks. As the

*We thank Roger Koenker, Steve Portnoy, Anil Bera, Qiwei Yao and two referees for very helpful comments on earlier versions of this paper.

bubble grows, the chances of it collapsing increases. The collapsing of bubbles leads to large negative returns and may therefore to negative skewness of returns since large positive returns are less likely. Finally, the time-varying probability of collapse may be linked to asymmetric volatility. To see this, note that rational investors may still invest in a stock even if they are aware that its price contains a collapsible bubble, as long as the bubble grows at a certain expected rate and they are able to sell at any time. If investors suddenly revise upward their subjective probability of a collapse, the conditional volatility of the stock return will increase, inducing asymmetric volatility.

Are there bubbles in stock prices? In the last two decades, there have been a number of studies on testing for speculative bubbles in stock prices, and different results have been obtained. For example, West (1987) and Rappoport and White (1993) found evidence of bubbles, while Diba and Grossman (1988) and Dezhbakhsh and Demirguckunt (1990) obtained results supporting the conclusion that stock prices do not contain bubbles. Of course, the presence of absence of bubbles has important implications for modeling stock prices and may have a serious impact on asset and risk management practices that use these models.

A popular approach in testing bubbles is to examine the stationarity (or non-stationarity) of the residuals between asset prices and market fundamentals [usually dividends, see Campbell and Shiller (1987), Diba and Grossman (1984, 1988), Hamilton and Whiteman (1985), among others, for applications based on this approach]. Traditionally this approach uses unit root tests on the residuals. By treating the nonstationary bubble process as a unit-root process, the conventional unit root based procedures test the null hypothesis that the stock price contains a bubble. If the unit root hypothesis is rejected, they reject the hypothesis of a bubble.

However, by conducting a Monte Carlo simulation, Evans (1991) showed that an important class of rational bubbles cannot be detected by the conventional unit root tests. In particular, he considered a time series of bubbles with a positive probability of collapse and found that the conventional unit root test over-reject the hypothesis of a bubble, except in the case where the probability of collapse is very close to zero.

One reason for this result is that collapsible bubbles are not conventional unit-root processes, so that the hypothesis of a bubble is not equivalent to the hypothesis of a unit 
root. If a bubble is collapsible, even when the market price contains a bubble, the conventional unit root based tests will still reject the null of a bubble, leading to the incorrect conclusion that there is no bubble in market prices. For this reason, the traditional unit-root tests have some serious drawbacks in detecting bubbles.

In this paper, we propose an alternative test for bubbles by looking at the fluctuations in the partial sum process of residuals from regressing asset prices on market fundamentals. In contrast to the conventional unit root based tests, the proposed test has its advantage in dealing with collapsible bubbles. Suppose that we consider the simple rational expectations model characterized by the Gordon model, then, intuitively, if there is no bubble, the stock price and the fundamentals move together in the long run. Therefore the residuals should be stationary, and the order of magnitude of the fluctuation in the cumulated sum process of residuals should be proportional to the square root of the number of observations. Otherwise, if there is a bubble, then the fluctuation in the cumulated bubble process should have much larger order of magnitude, regardless of the probability of collapse.

As noted by some researchers, since bubbles are not observable, no one can be absolutely certain they exist. At least at a casual level, evidence of excessive fluctuation of residuals between prices and fundamentals could result from sources other than bubbles. For example, time varying riskpremiums effects might be an alternative source of excessive fluctuation. In addition, covariance stationary time series with structural breaks may result in a sample path similar to that of a nonstationary time series. Flood and Hodrick (1986), Hamilton and Whiteman (1985) pointed out that bubbles may be observationally similar to regime changes in market fundamentals which are unobserved by researchers. Thus, evidence of nonstationarity do not necessarily establish the existence of bubbles. However, these tests can be used for converse inference. In particular, covariance stationarity in the residual process would be evidence against the existence of bubbles. As pointed out by Diba and Grossman (1988), except by extremely unlikely coincidence, other sources of nonstationarity could not exactly offset the effect of a nonstationary bubble.

The test statistic is constructed based on residuals from a cointegrating regression. In practice, the behavior of asset prices may be more complicated than that described by the Gordon model. To (at least partially) capture additional sources of fluctuation, we also augment the cointegrating regression of Campbell and Shiller (1987) (based the original Gordon model) by including additional covariates to take into account the time-varying discount rates and riskpremiums effects. Properties of the proposed tests are studied and some Monte Carlo experiments are conducted to investigate its performance. We believe that the test provides additional information on the behavior of the stock price that can not be fully explained by the underlying value of the firm. It also provides a useful alternative to the existing bubble tests and models of stock price dynamics.

This paper is organized as follows. Section 2 reviews the conventional testing procedure for rational bubbles based on the unit-root/cointegration approach. Section 3 develops the proposed test. Section 4 extends our analysis in the previous section to more general cases by including variables reflecting time-varying discount rates and risk-premiums. We conduct a simulation study in Section 5 and provide empirical application of the test to U.S. and Hong Kong stock indexes in Section 6. Some technical details are included in the Appendix.

\section{CONVENTIONAL TESTING PROCEDURES FOR BUBBLES}

In this paper, we consider a standard rational expectations model for stock prices

$$
P_{t}=(1+\gamma)^{-1} E_{t}\left(P_{t+1}+D_{t+1}\right)
$$

where $P_{t}$ is the real stock price at $t, \gamma$ is the real rate of return and thus $(1+\gamma)^{-1}$ is the discount factor, and $D_{t}$ is the dividend paid to the owner of the stock between $t$ and $t+1$. Equation (1) is a first-order expectational difference equation. A forward-looking solution to this equation can be obtained by straightforward calculation, and a test for the presence of bubbles can be constructed based on a regression of $P_{t}$ on $D_{t}$ (plus a constant) (see, inter alia, Diba and Grossman (1988), Evans (1991)).

However, there has been concern about the above specification: violation of limited liability. For instance, if the conditional distribution of the prices is normal, then there will always be a positive probability of obtaining a negative price (see, e.g., Campbell, Lo and MacKinlay (1997, p. 32)). For this reason, many researchers consider the above rational expectations model in terms of logarithms of price and dividend. Following Campbell and Shiller (1988), we write the log linear approximation of (1) as

$$
p_{t}+q=\kappa+\delta E_{t} p_{t+1}+(1-\delta) E_{t} d_{t+1}
$$

where $p_{t}$ and $d_{t}$ are logarithms of $P_{t}$ and $D_{t}, q$ is the log gross return rate, $\delta$ is the average ratio of the stock price to the sum of the stock price and the dividend $(0<\delta<1)$, and $\kappa$ is a function of $\delta$ (see Wu (1997) for a discussion of this model). Under the transversality condition that

$$
\lim _{k \rightarrow \infty} \delta^{k} E_{t} p_{t+k}=0,
$$

the unique forward-looking market fundamental solution to $(2), f_{t}$, is given by

$$
f_{t}=\eta+(1-\delta) \sum_{j=0}^{\infty} \delta^{j} E_{t} d_{t+1+j} .
$$


If the transversality condition (3) does not hold, the general solution to (2) has the following form

$$
p_{t}=f_{t}+b_{t},
$$

where $f_{t}$ is the market fundamental given by (4) and $b_{t}$ is a rational speculative bubble generated by extraneous events or rumors and satisfies

$$
E_{t} b_{t+1}=\frac{1}{\delta} b_{t} .
$$

By definition, this bubble grows over time, driven by selffulfilling expectations. However, the bubble must eventually collapse in order for the model to be plausible.

Remark 1. Notice that there is a literature on the debate of whether rational bubbles can exist. For example, Diba and Grossman (1988) argue that any rational bubble that starts after the first date of trading has an expected initial value of zero. The fact that rational bubbles have explosive conditional expectations implies that a negative-bubble cannot exist on an asset with limited liability, thus the expected initial value can equal zero only if any initial realization of a rational bubble after the first date of trading equals zero with probability one. Also see Tirole $(1982,1985)$ for study of bubbles in general equilibrium models and overlappinggenerations models, and Cambell, Lo and MacKinlay (1997, Section 7.1.2) for an excellent literature review on this topic. This is a continuing debate and is not the focus of the current paper.

Since $d_{t}$ appears to be nonstationary in empirical analyses, it is usually characterized as an integrated process with drift:

$$
d_{t+1}=\mu+d_{t}+\varepsilon_{t},
$$

where $\varepsilon_{t}$ is an $\mathrm{I}(0)$ process of innovations with $E\left(\varepsilon_{t}\right)=0$. Combining (4) and (7), we have

$$
f_{t}=\alpha+\beta d_{t},
$$

and (5) can be written as

$$
p_{t}=\alpha+\beta d_{t}+b_{t} .
$$

If there is no bubble, $p_{t}=f_{t}$, which can be expressed as (8), thus $p_{t}$ is also an integrated process with drift. Although both $p_{t}$ and $d_{t}$ are nonstationary, there exists a long run equilibrium relationship between $p_{t}$ and $d_{t}$, and the linear combination of $p_{t}$ and $d_{t}\left(p_{t}-\alpha-\beta d_{t}\right)$ is $\mathrm{I}(0)$. Fluctuations in the residual process $p_{t}-\alpha-\beta d_{t}$ are simply equilibrium errors and thus are covariance stationary. In other words, $p_{t}$ and $d_{t}$ are cointegrated in the absence of bubbles. Otherwise, if the stock price contains a bubble, $p_{t}$ and $d_{t}$ are no longer cointegrated since the bubble process is not covariance stationary given that $E_{t} b_{t+1}=\delta^{-1} b_{t}$ and $\delta^{-1}>1$.
Much of the literature on testing for bubbles uses the residual-based tests for cointegration of Engle and Granger (1987) and Phillips and Ouliaris (1991). These tests consider the null hypothesis of no cointegration and apply the traditional unit-root tests to the least square residuals from a regression of the stock prices on the dividends. Thus, the procedures test the null hypothesis that the residual process of (9) contains a unit-root. By treating the bubble process as a unit-root process, they test the null hypothesis that the stock price contains a bubble.

The approach is typically done by first applying unitroot tests to $p_{t}$ and $d_{t}$ to verify their nonstationarity. Then, a cointegrating regression between $p_{t}$ and $d_{t}$,

$$
p_{t}=\widehat{\alpha}+\widehat{\beta} d_{t}+\widehat{u}_{t},
$$

is performed to obtain the residual process $\widehat{u}_{t}=p_{t}-\widehat{\alpha}-\widehat{\beta} d_{t}$. The existence of a bubble is then tested by applying the unitroot tests on the residuals $\widehat{u}_{t}$. If the residual process contains a unit-root, then $p_{t}$ and $d_{t}$ are not cointegrated, and thus the conclusion that the stock prices contain a bubble is obtained. If $\widehat{u}_{t}$ does not have a unit-root, we reject the null hypothesis and conclude that there is no bubble in the price series.

These testing procedures rely on unit-root tests (usually ADF or PP tests) which were designed for detecting whether or not a time series contains an autoregressive unit-root. However, the capability of these procedures to detect bubbles depends on the assumption that the bubble process can be characterized by an autoregressive process with a constant coefficient around unity (or larger than unity). If a bubble collapses and restarts with a certain probability, then this assumption no longer holds. For example, if the representation of bubbles is characterized by an autoregressive $(\mathrm{AR})$ process as

$$
b_{t+1}=\alpha b_{t}+u_{t+1}, \quad \alpha=1 / \delta,
$$

where $u_{t}$ is stationary, then the residual based tests for the null hypothesis of no cointegration are effective for testing bubbles. However, it is not necessary that a bubble should be characterized by the AR process (11). In fact, it should be able to collapse after reaching high levels and then restart. For example, a simple model of periodically collapsing bubbles may be characterized as follows,

$$
b_{t+1}=a_{t+1} b_{t}+u_{t+1},
$$

where $u_{t}$ is a mean zero stationary residual process and $a_{t+1}$ is an exogenous independently and identically distributed (i.i.d.) Bernoulli process which takes value $a$ with probability $\pi$ and value 0 with probability $1-\pi$. When $a=1 /(\delta \pi)$, $E_{t}\left(b_{t+1}\right)=\delta^{-1} b_{t}$, then $b_{t}$ is a rational bubble [see Tirole (1982)].

The process $\left\{b_{t}\right\}$ determined by (12) is a doubly stochastic process (for studies on stationarity property of this type process, see, e.g., Nicholls and Quinn (1982), Pourahmadi 
(1988)). In particular, a process defined by (12) is covariance stationary if $\operatorname{Var}\left(a_{t}\right)+\left[E a_{t}\right]^{2}<1$. In the case that $b_{t}$ is a rational bubble, $E a_{t}>1$ and thus $\operatorname{Var}\left(a_{t}\right)+\left[E a_{t}\right]^{2}>1$. The process is no longer covariance stationary and the variance of $b_{t}$ diverges to infinity exponentially as $t$ increases.

However, although this process is not covariance stationary, it is not a conventional unit-root process. Rather, it is a random coefficient process. As a result, the commonly used unit-root tests are not valid procedures for detecting the existence of such a process.

Other types of collapsible bubbles can be designed. Although difference exists among different types of bubbles, as long as they are rational bubbles, they satisfy the property that $E_{t}\left(b_{t+1}\right)=\delta^{-1} b_{t}$ and thus are not covariance stationary. In addition, if they collapse, they are not conventional unit root process and can not be detected by traditional unit root tests. A good example of reporting that conventional unit root tests are unable to detect collapsible bubbles is in Evans (1991, page 926, right column, first complete paragraph onwards). In particular, Evans (1991) considered a multiplicative process with periodical collapsible bubbles and found that the unit root tests can hardly detect a collapsible bubble.

\section{A TEST FOR SPECULATIVE BUBBLES}

In this section, we suggest an alternative way to test the null hypothesis of no bubble in $p_{t}$. Again, we start with the model given by (5) and (8) in Section 2, and consider the residual process from the following regression

$$
p_{t}=\alpha+\beta d_{t}+u_{t}
$$

As discussed in Section 2, if there is no bubble in the stock price, the residual term $u_{t}$ in (13) should be covariance stationary, and fluctuations in this process are just equilibrium errors. In particular, the order of magnitude of the partial sum process $\sum_{t=1}^{k} u_{t}$ should be proportional to $\left(k^{1 / 2}\right)$. Under mild conditions $n^{-1 / 2} \sum_{t=1}^{[n r]} u_{t}(0<r<1)$ satisfies an invariance principle.

On the other hand, if there is a bubble in the stock price, then the residual term $u_{t}$ includes a bubble process $\left\{b_{t}\right\}$. Even though in each period there is a positive probability that $b_{t}$ will collapse (and the bubble will eventually explode), by the defining property of a rational bubble that $\left|E_{t} b_{t+1}\right|>\left|b_{t}\right|$, the variance of $b_{t}$ (and thus the residual term $u_{t}$ of regression (13)) is increasing with $t$ exponentially! Consequently $b_{t}$ is not $\mathrm{I}(0)$. In particular, the fluctuation in the residuals is dominated by the bubble component and the cumulated sums of $u_{t}$ will have a much larger order of magnitude than the no bubble case. In fact, $\sum_{t=1}^{k} u_{t}$ diverges to $\infty$ exponentially. This observation suggests that it is possible to design a bubble test by looking at the order of magnitude of the fluctuation in the residual process.

We consider the following quantity as a measurement of fluctuation in the residual process $\widehat{u}_{t}$ [Sen (1980), Xiao
(2001)], where $\widehat{u}_{t}$ is the estimated residual process from a cointegrating regression of (13),

$$
\max _{k=1, \ldots, n} \frac{k}{\sqrt{n}}\left|\frac{1}{k} \sum_{t=1}^{k} \widehat{u}_{t}-\frac{1}{n} \sum_{t=1}^{n} \widehat{u}_{t}\right| .
$$

This is the recursive-estimates statistic for testing fluctuation. As discussed above, in the case with no bubbles, the order of magnitude of $\max _{k} \sum_{t=1}^{k} u_{t}$ is $O_{p}(\sqrt{n})$. Standardized by $\sqrt{n}, \max _{k=1, \ldots, n} \frac{1}{\sqrt{n}}\left|\sum_{t=1}^{k} u_{t}\right|$ converge to a Brownian motion. In the presence with bubble, $\max _{k=1, \ldots, n} \frac{1}{\sqrt{n}}\left|\sum_{t=1}^{k} u_{t}\right|$ diverge to $\infty$, providing a potential test. Notice that property that a Brownian motion always has very small variance close to the origin and large variance when $r$ is close to 1 , we tie down the process by subtracting it by $\frac{1}{\sqrt{n}}\left(\frac{k}{n} \sum_{t=1}^{n} \widehat{u}_{t}\right)$, giving a Brownian bridge limit. Similar statistics can also be used to test the structural stability in linear regression models (e.g., Ploberger, Kramer and Koutrus (1989)). While the structural stability literature focuses on the constancy of the regression parameter, we focus on the residuals and consider a test for the null hypothesis of no bubbles against the alternative of bubbles. As will be clear in later analysis, these two models (structural break in parameters and collapsing bubbles) have similar behavior under the null hypotheses but are different under the alternatives (see the Appendix for analysis under the alternative). In particular, these two models have different fluctuations under the alternatives, although (14) diverges in both models. In the structural break models, the stationarity property of the true residuals does not change, and the fluctuation comes from the break in regression parameter. In our model, the residual process is no longer covariance stationary under the alternative and has an increasing (explosive) variance.

Notice that $d_{t}$ is nonstationary $(\mathrm{I}(1))$ and $d_{t}$ and $u_{t}$ may be correlated. As a result, the limiting distribution of the recursive-estimates statistic is dependent on nuisance parameters coming from the serial correlation of $d_{t}$ and $u_{t}$ and on the correlation between these two series. Thus, this statistic cannot be directly used in testing. To get rid of the nuisance parameters, we construct the fully modified residual process $\widetilde{u}_{t}^{+}$based on nonparametric estimation of the nuisance parameters as follows:

$$
\widetilde{u}_{t}^{+}=p_{t}^{+}-\left(1, d_{t}\right)\left(\begin{array}{c}
\widehat{\alpha}^{+} \\
\widehat{\beta}^{+}
\end{array}\right)=p_{t}^{+}-\widehat{\alpha}^{+}-\widehat{\beta}^{+} d_{t},
$$

where $\widehat{\alpha}^{+}$and $\widehat{\beta}^{+}$are the fully modified estimators of $\alpha$, $\beta, p_{t}^{+}=p_{t}-\Delta d_{t}^{\prime} \widehat{\Omega}_{d d}^{-1} \widehat{\Omega}_{d u}$ are the fully modified prices, and $\widehat{\Omega}_{d d}$ and $\widehat{\Omega}_{d u}$ are nonparametric estimators of the long-run variance of $d_{t}$ and of the long-run covariance between $d_{t}$ and $u_{t}$. The definition of the fully modified estimators and the construction of nonparametric estimators can be found in Phillips and Hansen (1990), Phillips (1995), and Xiao 
and Phillips (2002), and we give them in the Appendix for completeness.

Based on the fully modified residual process $\widetilde{u}_{t}^{+}$, we construct the test statistic as follows,

$$
\underline{R}=\max _{k=1, \ldots, n} \frac{k}{\widehat{\omega}_{u . d} \sqrt{n}}\left|\frac{1}{k} \sum_{t=1}^{k} \widetilde{u}_{t}^{+}-\frac{1}{n} \sum_{t=1}^{n} \widetilde{u}_{t}^{+}\right|,
$$

where $\widehat{\omega}_{u . d}^{2}$ is the nonparametric long-run variance estimator [We give the formula of this estimator and related discussions in the Appendix]. As the sample size $n$ goes to infinity, the test statistic (15) has the classical Kolmogoroff-Smirnoff type limiting distribution

$$
\sup _{0 \leq r \leq 1}|\widetilde{V}(r)|,
$$

where $\tilde{V}(r)=\underline{W}_{d}(r)-r \underline{W}_{d}(1), \underline{W}_{d}(r)=W_{1}(r)-$ $\left[\int_{0}^{1} d W_{1} S^{\prime}\right]\left[\int_{0}^{1} S S^{\prime}\right]^{-1} \int_{0}^{r} S, S(r)^{\prime}=\left(1, W_{2}(r)\right)$, and $W_{1}(r)$ and $W_{2}(r)$ are standard Brownian motions that are independent of each other. The limiting distribution of this test is free of nuisance parameter. We computed the relevant upper tail critical values for the asymptotic null distribution of $\underline{R}$ based on a direct simulation using a sample of size 2,000 and 20,000 replications and report them in the following table:

\begin{tabular}{lcccccccc}
\hline Critical & $0.5 \%$ & $1 \%$ & $1.5 \%$ & $2 \%$ & $2.5 \%$ & $3 \%$ & $3.5 \%$ & $4 \%$ \\
$\begin{array}{l}\text { Level } \\
\text { Critical }\end{array}$ & 1.544 & 1.448 & 1.363 & 1.325 & 1.295 & 1.267 & 1.242 & 1.218 \\
Value & & & & & & & & \\
\hline $\begin{array}{l}\text { Critical } \\
\text { Level }\end{array}$ & $4.5 \%$ & $5 \%$ & $6 \%$ & $7 \%$ & $8 \%$ & $9 \%$ & $10 \%$ & $15 \%$ \\
$\begin{array}{l}\text { Critical } \\
\text { Value }\end{array}$ & 1.198 & 1.176 & 1.141 & 1.115 & 1.085 & 1.065 & 1.047 & 0.974 \\
\hline
\end{tabular}

Remark 2. The proposed test has power against bubbles not because of the "breaks" (collapses) of bubbles (at least asymptotically), but because of the explosive behavior determined by the defining property (6) of rational bubbles! The proposed test has power against both collapsible bubbles and non-collapsible (if there is any) bubbles. The "breaks" (collapse) in the bubble processes in this paper is different from the conventional structural breaks. Actually, for the process $\left\{b_{t}\right\}$ defined as (12), if $\operatorname{Var}\left(a_{t}\right)+\left[E a_{t}\right]^{2}$ were strictly less than 1 , even in the presence of collapse, the process $b_{t}$ would still be covariance stationary and the proposed test will not detect it in large sample.

To be effective, a statistical test must discriminate between the null and the alternative in large samples. This is a nontrivial matter for the proposed test because both the numerator and the denominator of the test statistic diverge under the alternative. The order of magnitude of the proposed test statistic is determined by the partial sum process

$$
\frac{1}{\sqrt{n}} \sum_{t=1}^{n} \widetilde{u}_{t}^{+}
$$

and the nonparametric long-run variance estimator $\widehat{\omega}_{u . d}$ that enters the denominator. Under the alternative hypothesis that there is a bubble in the stock price, both of these two quantities will diverge to infinity. In particular, it can be shown that (17) diverges to $\infty$ exponentially at rate $\sqrt{\Delta^{n} / n}$, where $\Delta=\sigma_{a}^{2}+1 / \delta^{2}>1$, and $\sigma_{a}^{2}$ is the variance of the random coefficient in the bubble process (if the bubble does not collapse, $\sigma_{a}^{2}=0$ and $\Delta=1 / \delta^{2}$, which is still greater than 1). Under $H_{1}$, the nonparametric kernel estimator $\widehat{\omega}_{u . d}$, constructed from the residuals, diverges as well. The divergence rate of $\widehat{\omega}_{u . d}$ is dependent on the sample size that used in the calculation of $\widehat{\omega}_{u . d}$, and the bandwidth (lag truncation) choice. In order to preserve consistency, we need to construct the long-run variance estimator appropriately so that the denominator $\left(\widehat{\omega}_{u . d}\right)$ diverges at a slower rate than (17). In particular, if we use a subsample of size $m$ and a bandwidth $M$ to estimate $\widehat{\omega}_{u . d}$, (both $M$ and $m$ go to infinity with $n$ ), it can be shown that, in the presence of a bubble, the order of magnitude is $O_{p}\left(\sqrt{M \Delta^{m} / m}\right)$. Under the conditions that $n-m \rightarrow \infty$, and $M \rightarrow \infty$ at a lower rate than $m$, it is easy to verify that under $H_{1}$, the test statistic diverge to $\infty$, giving a consistent test (see the Appendix for more discussion on this).

As mentioned before, similar statistics can be used in testing structural stability in linear regression models and the test (15) also has power against structural break (or breaks). However, we also note that these two types of models have different types of fluctuation under the alternatives. In particular, in the structural break model, the fluctuation comes from the break in regression parameter. Such breaks only happen in finite times (usually assumed once in many typical models). If the location of a break point is known, this maybe the case if there are known interventions or policy changes, the structural break can be characterized using appropriate dummy variables. Given the types of bubbles that we consider in this paper, it is possible to construct a test for the null hypothesis of no bubble (but with potential structural breaks) against the alternative of bubbles. Of course, we need to specify the model in this case to obtain a valid test. For example, we may consider the following model that, under the null hypothesis, there is a possible structural break at time $t=n_{0}=\tau n$, where $0<\tau<1$, and the null model with a structural break is characterized as

$$
p_{t}=\alpha+\beta_{t} d_{t}+u_{t}, \quad t=1,2, \ldots, n,
$$

where

$$
\beta_{t}= \begin{cases}\beta_{1}, & t=1,2, \ldots, n_{0} \\ \beta_{2}, & t=n_{0}+1, \ldots, n,\end{cases}
$$

and $u_{t}$ is a covariance stationary time series. Under the alternative, the asset price, and thus $u_{t}$, contains a bubble and

$$
p_{t}=\alpha+\beta_{t} d_{t}+b_{t}, \quad t=1,2, \ldots, n .
$$


Then, defining a dummy variable

$$
h_{t}= \begin{cases}0, & t=1,2, \ldots, n_{0} \\ 1, & t=n_{0}+1, \ldots, n\end{cases}
$$

we may write the model as

$$
p_{t}=\alpha+\beta d_{t}+\theta h_{t} d_{t}+u_{t}, \quad t=1,2, \ldots, n .
$$

Noticing the difference between the bubbles and the structural break that we consider in this paper, the dummy variables can capture the property of a structural break, but not bubbles. If we regress $p_{t}$ on a constant term, the dividend $d_{t}$, and $h_{t} d_{t}$, a residual based test can still be constructed as before. In particular, the partial sum process $\frac{1}{\widehat{\omega}_{u . d} \sqrt{n}} \sum_{t=1}^{[n r]} \widetilde{u}_{t}^{+}$ will now converge to a limiting process $\check{V}_{\tau}(r)$ defined on an underlying Wiener process that will be dependent on the number of regressors (which is 2 here) and $\tau$. The asymptotic distribution of the test then has a similar form as (16). We may compute the critical values of the test for different values of $\tau$ by simulating empirical approximations to Brownian motion with a large sample size and replications. As $\tau \rightarrow 0$, or 1 , the limiting distribution and critical values will approach to those with no break. As anticipated, introducing a structural break reduces the power of the test.

Notice that to distinguish the bubbles from structural breaks, it is necessary to specify the bubble and breaks. Without enough specification about the bubble and breaks, or, with incorrect specification of the structural break, it is very difficult to distinguish between structural breaks and bubbles.

\section{GENERALIZATIONS}

In Section 3 we used the model of Campbell and Shiller (1987) [also see, e.g., Gordon (1962), Evans (1991), among others, for the use of this type models] where the market fundamental price is characterized by (4). Indeed, to our knowledge, the cointegration based approach of testing bubbles has been exclusively focused on this type of models with a constant rate of return. Relaxing the assumption of a constant rate of return will substantially complicate the forward-looking solution to the rational expectation model. In general, there is no simple analytical solution to the market fundamental price unless we impose additional assumptions on the associated conditional expectation.

Besides dividends, other sources of stock price fluctuations may be accounted for. As mentioned in the introduction, time-varying discount rates and risk-premium effects may all be possible sources of excessive fluctuations. Consequently, covariates that can help explain market fundamentals may also be included in regression (13). However, it is not easy to construct a general model that fits all the stylized facts given in the literature. The above mentioned generalizations substantially complicate the market fundamental solution, appropriate simplification of the market fundamental solution has to be introduced. In this section, we attempt to provide a first step toward incorporating other market fundamental sources into the cointegration based approach.

\subsection{Time-varying discount rate}

As an alternative of (1), we may consider a more general model which allows $\gamma$ change over time:

$$
P_{t}=E_{t}\left\{\frac{P_{t+1}+D_{t+1}}{1+\gamma_{t}}\right\} \text {. }
$$

Solving Equation (18) recursively and denoting the growth rate of real dividend as $g_{t}$, we obtain the following expression for the fundamental value of asset prices:

$$
F_{t}=E_{t}\left\{\sum_{j=0}^{\infty}\left(\prod_{i=0}^{j}\left[\frac{1+g_{t+i}}{1+\gamma_{t+i}}\right]\right)\right\} D_{t} .
$$

Gordon (1962) assumes that $\gamma$ and $g$ are constant and thus (19) reduces to

$$
F_{t}=D_{t}\left(\frac{1+g}{\gamma-g}\right)
$$

which delivers the regression model we considered in Section 3. In the general case, if we denote the price-dividend ratio as

$$
\phi_{t}=\log E_{t}\left\{\sum_{j=0}^{\infty}\left(\prod_{i=0}^{j}\left[\left(1+g_{t+i}\right) /\left(1+\gamma_{t+i}\right)\right]\right)\right\},
$$

then $f_{t}$ can be expressed as a linear function of $d_{t}$ and $\phi_{t}$. If $\phi_{t}$ were known, we could consider the residual process from the following regression

$$
p_{t}=\alpha_{0}+\beta_{0} d_{t}+\lambda_{0} \phi_{t}+u_{t}
$$

and construct a residual based test for bubbles as in Section 3. However, $\phi_{t}$ is unknown and appropriate approximation of $\phi_{t}$ has to be used. Giving assumptions about $g_{t}$ and $\gamma_{t}$, we may approximate this time-varying parameter. For example, Barsky and DeLong (1993) considered the relationship (20) and assume that $g$ in (20) is nonconstant and replace it by a (geometrically averaged) function of $g_{t-j}$, $j=0,1, \ldots, k$. Donaldson and Kamstra (1996) use a similar idea in estimating market fundamental and conduct an empirical analysis of bubbles by using unit root tests on the difference between the asset price and the estimated market fundamental.

Notice that $\phi_{t}$ is the expectation of the price-dividend ratio conditional on the information set at time $t$. If certain variables, say $z_{t}$, contain past information about $\gamma$ and $g$, $\phi_{t}$ is also a function of $z_{t}$ and we may consider using $z_{t}$ (or functions of $z_{t}$ ) as an instrument of $\phi_{t}$. For example, the 
Capital Asset Pricing Model (CAPM) shows that the total return to equity (the sum of capital gains and the dividend yield) as linearly related to the interest rate. Based on the CAPM, a reasonable instrument for the price-dividend ratio (which is the inverse of the dividend yield) might be the inverse of the short term interest rate $r_{t}$. We denote the inverse of the short term interest rate $r_{t}$ as $z_{t}$. For simplicity, if we assume that $\phi_{t}$ is a linear function of $z_{t}$, say $\phi_{t}=$ $\beta_{1}+\beta_{2} z_{t}$, then we obtain

$$
p_{t}=\left(\alpha_{0}+\lambda_{0} \beta_{1}\right)+\beta_{0} d_{t}+\lambda_{0} \beta_{2} z_{t}+u_{t} .
$$

The above representation suggests that if we consider the following regression

$$
p_{t}=\widehat{\alpha}+\widehat{\beta} d_{t}+\widehat{\lambda} z_{t}+\widehat{u}_{t},
$$

we may test the existence of bubbles by looking at the fluctuation in the residual process $\widehat{u}_{t}$, and thus a test similar to $(15)$ can be constructed. Denote

$$
\Pi=\left[\begin{array}{ccc}
\alpha, & \beta, & \lambda
\end{array}\right], \quad \text { and } \quad w_{t}^{\prime}=\left[\begin{array}{lll}
1, & d_{t}, & z_{t}
\end{array}\right],
$$

and for the same reason as in Section 3, we construct the fully modified residual process

$$
\widehat{u}_{t}^{+}=p_{t}^{+}-\widehat{\Pi}^{+} w_{t},
$$

where $\bullet+$ signifies fully modification (thus $\widehat{\Pi}^{+}$is the fully modified estimate of $\Pi$ ). A test for the null hypothesis of no bubbles can be constructed by replacing $\widetilde{u}_{t}^{+}$in (15) by $\widehat{u}_{t}^{+}$. Under the null hypothesis of no bubble, as $n \rightarrow \infty$, the corresponding test statistic has a limiting distribution similar to (16), but with $S(r)^{\prime}=\left(1, W_{2}(r)^{\prime}\right), W_{1}(r)$ and $W_{2}(r)$ are 1 and 2-dimensional standard Brownian motions that are independent of each other.

\subsection{Time-varying risk-premiums}

Time-varying risk premium provides another source of fluctuation in stock prices. Campbell and Cochrane (1999) considered a simple model with a representative agent whose utility displays habit-formation. Such a utility function makes the agent more risk-averse in bad times, when consumption is low relative to its past history, than in good times, when consumption is high relatively to its past history. Campbell and Cochrane find that a good account of fluctuations in stock prices is a time varying (countercyclical) risk premium. Their finding suggests that variables measuring time-varying risk premium are useful covariates to explain the behavior of prices. For this reason, we augment the cointegrating regression (21) with a covariate $A A A_{t}-B B B_{t}$ (the difference of the yields-to-maturity on high-grade and low-grade bonds) and consider the following regression ${ }^{1}$

$$
p_{t}=\widehat{\alpha}+\widehat{\beta} d_{t}+\widehat{\lambda} z_{1 t}+\widehat{\delta} z_{2 t}+\widehat{u}_{t}=\widehat{\theta}^{\prime} x_{t}+\widehat{u}_{t},
$$

\footnotetext{
${ }^{1}$ We thank a referee for this helpful suggestion.
}

where $z_{1 t}$ is the inverse of the short term interest rate and $z_{2 t}=A A A_{t}-B B B_{t}$, and

$$
x_{t}^{\prime}=\left(1, d_{t}, z_{1 t}, z_{2 t}\right) ; \quad \theta^{\prime}=(\alpha, \beta, \lambda, \delta) .
$$

The test can be constructed in a similar way to (15). Under the null hypothesis of no bubble, as $n \rightarrow \infty$, the corresponding test statistic has a limiting distribution similar to (16), but with $S(r)^{\prime}=\left(1, W_{2}(r)^{\prime}\right)$, where $W_{2}(r)$ is a 3 dimensional standard Brownian motion and is independent of $W_{1}(r)$.

It is well-known that conditional heteroskedasticity (such as ARCH (Engle (1982)) or GARCH (Bollerslev (1987)) effects) can bring excess fluctuation to the residuals. However, the fluctuation brought by ARCH or GARCH effects is different from the fluctuation brought by bubbles in order of magnitude. These two types of fluctuations can be distinguished by the proposed tests. In particular, although the conditional heteroskedastic residuals brings fluctuation to the prices, the order of magnitude of the cumulated sums is still proportional to the square root of the number of observations, while the fluctuation in the cumulated bubble process have larger order of magnitude.

Consequently, in the case that the residual term $u_{t}$ is conditional heteroskedastic, the limiting null distribution of the test statistic is still given by (16), and the result of our previous analysis are asymptotically robust to $\mathrm{ARCH}$ or $\mathrm{GARCH}$ effects. The proposed tests can not discriminate between the hypotheses "bubble-free with constant" and "bubble-free with conditional heteroskedasticity", but can discriminate between the hypotheses "bubble-free with conditional heteroskedasticity" and "process with bubble". This is because that the invariance principle (of the partial sum process) are robust to heteroskedasticity (see, Pantula (1986, 1988), Peters and Veloce (1988), Phillips (1987), Kim and Schmidt (1993) for related studies). For this reason, the proposed test in Section 4.1 can also be used in testing the null hypothesis of "bubble-free with conditional heteroskedasticity" against the alternative of "bubble". The test proposed in Section 4.2 can also be used in testing the null hypothesis of "bubblefree with time-varying risk premiums and conditional heteroskedasticity" against the alternative of "bubble".

Remark 3. If we could impose the restriction of "no bubble" in our estimation procedure, we could generate (by Monte Carlo experiment) the critical values using the estimated parameters. Unfortunately, we do not know whether or not there is a bubble in the data and it is difficult to restrict the model to be "no bubble" in estimation. Consequently, if we generate the critical values by, say, bootstrapping the data, the test will be inconsistent because it would also capture the behavior under the alternative.

\section{A SIMULATION STUDY}

In this section, we conduct a simulation study to evaluate the proposed test and provide a comparison with the traditional unit root test-based procedures. As we mentioned in 
the previous sections, both the proposed procedure and the unit root test based procedures can also be used for other inference problems than bubble tests. Our model differs from the previous models under the alternative hypothesis with bubbles. Under the hypothesis of no bubbles, the model that we study in this paper will be the same as other models (on structural stability and unit roots) that have been examined in the previous literature. For this reason, the Monte Carlo study in this section focuses on the behavior of the tests in the presence of bubbles, although we also present some results of the empirical size for completeness. For more discussion on their behavior under the hypothesis of no bubbles, see, for example, Schwert (1989); and Xiao and Phillips (2002).

In generating time series data, we try to calibrate the realworld data. In particular, we resample the dividend series from the weekly Standard and Poor 500 Index from January 1974 to September 1998 in each iteration. Notice that the dividend data are temporally dependent and nonstationary. To preserve these properties in the generated data, we consider a sieve bootstrap for the differenced dividend series. The idea of sieve resampling for a time series is as follows: a weakly dependent time series can be approximated by a sequence of autoregressive process of order $p=p(n)$, where $p(n)$ increases with the sample size. In practice, we may use criteria such as BIC or AIC for the selection of $p$. In our simulation, we use the BIC criterion in selecting $p$. We resample the dividend series based on the following steps: Denoting the original dividend time series as $\left\{D_{t}\right\}_{t=1}^{T}$ and let $w_{t}=\Delta D_{t}$, we fit the approximated autoregression

$$
w_{t}=\widehat{\rho}_{1} w_{t-1}+\cdots+\widehat{\rho}_{p} w_{t-p}+\widehat{\varepsilon}_{t}
$$

by the usual OLS regression. We center $\widehat{\varepsilon}_{t}$ and denote the centered innovations as $\widetilde{\varepsilon}_{t}$ and then draw an i.i.d. sample $\left\{\varepsilon_{t}^{*}\right\}$ from $\left\{\widetilde{\varepsilon}_{t}\right\}$. Generate $\left\{w_{t}^{*}\right\}$ from $\left\{\varepsilon_{t}^{*}\right\}$ by

$$
w_{t}^{*}=\widehat{\rho}_{1} w_{t-1}^{*}+\cdots+\widehat{\rho}_{p} w_{t-p}^{*}+\varepsilon_{t}^{*}, \quad t=k+1, \ldots, T,
$$

with $w_{j}^{*}=w_{j}$, for $j=1, \ldots, p$, and finally obtain the dividend resample $\left\{D_{t}^{*}\right\}$ by

$$
D_{t}^{*}=D_{t-1}^{*}+w_{t}^{*}, \quad t=2, \ldots, T,
$$

with $D_{1}^{*}=D_{1}$. For asymptotic properties of the sieve bootstrap procedures, see, inter alia, Buhlmann (1997), Bickel and Buhlmann (1998), Chang and Park (2000).

Next, we generate data for prices. In the presence of bubbles, prices are determined by market fundamentals and bubbles. However, since we don't know if bubbles exist, we can not just simply resample the real-world price series. Instead, we have to simulate bubbles. The generated price series is then the sum of the simulated bubble and the generated market fundamental.

We generate the bubble process based on (12) where the residual terms $u_{t}$ in the bubble process are taken from independent student- $t$ distributions with 4 degrees of freedom. The initial value of $b_{t}$ is set at $b_{0}=0$ and we choose $\delta=0.9090$, since the sample average market return in the past 10 years is about $10 \%$. For probability $\pi$, we consider $\pi=0.9,0.93,0.95,0.96,0.98$, corresponding to a probability of collapse of $0.1,0.07,0.05,0.04$, and 0.02 .

Based on the dividend series $\left\{D_{t}^{*}\right\}$, we generate the market fundamental process using the Gordon fundamental pricing formula (20). ${ }^{2}$ Again, we need to choose the values of parameters $\gamma$ and $g$. In our Monte Carlo experiment, we choose $\gamma$ to be 0.1 and $g$ to be 0.04 . These are the long run discount rate and dividend growth rate. Finally, the prices (with bubbles) are obtained by adding the generated bubble processes to the generated market fundamentals.

To examine the size of the test $\underline{R}$, we generate the prices by adding a stationary component to the generated market fundamentals. In our Monte Carlo experiment, we consider three simple cases: an i.i.d. process of $\mathrm{N}(0,1)$ random variables and two $\mathrm{AR}(1)$ processes with different values of $\mathrm{AR}$ coefficients $u_{t}=\rho u_{t-1}+\varepsilon_{t}$, with $\rho=0.2$ and 0.8 respectively.

We compare the proposed test $\underline{R}$ with the traditional unit root based procedures (the augmented Dickey-Fuller and Phillips-Perron t-ratio tests) that have been used in bubble testing. The lag length in the ADF test is chosen based on the BIC criterion. For the proposed test $\underline{R}$, since it is based on nonparametric estimators we need to choose bandwidth parameters, we calculate the test statistics for a number of representative values of the bandwidth parameter $M$ and the subsample parameter $m$. In particular, we consider the following bandwidth choices: $M 1=\left[4(\mathrm{~m} / 100)^{1 / 4}\right]$, $M 2=\left[6(m / 100)^{1 / 4}\right]$, and $M 3=\left[8(m / 100)^{1 / 4}\right]$. For the subsample size in the nonparametric estimation, we consider $m=\left[n^{0.9}\right],\left[n^{0.8}\right]$. All tests are conducted at the $5 \%$ critical level. Four different sample sizes are examined. The sample sizes in each iteration are $n=200,300,500$, and 900. The number of iterations is 400 in each case of data generation. Table 1 and Table 2 report the empirical size and power of the proposed test $\underline{R}$, and Table 3 provides the simulation results of the traditional unit root based procedures.

From Table 1, we can see that the test has reasonable size if the bandwidth is appropriately chosen. In the case with i.i.d. residuals, a short bandwidth is appropriate. The test under-rejects when the bandwidth value is large. For the case of AR residuals with $\rho=0.8$, the bandwidth choice $M 3$ works best since the serial correlation in the residuals is pretty high and thus a large bandwidth is needed to capture the dependence in the residuals.

The Monte Carlo results contained in Table 3 indicate that the ability of the unit root tests to detect violations of the null of a bubble is low when it is collapsible. Both the ADF test and the Phillips-Perron test tend to over-reject the null hypothesis of a bubble when the bubble is collapsible, leading to the incorrect conclusion that there are no bubbles.

${ }^{2}$ For simplicity, we make use of the Gordon model in our experiment as an approximation here. A more realistic series would be generated by using a serially correlated process of growth rates. 
Table 1. Empirical size of the test $\underline{R}$

\begin{tabular}{|c|c|c|c|c|c|}
\hline \multicolumn{6}{|c|}{ I.I.D. residuals } \\
\hline \multirow{4}{*}{$m=n^{0.8}$} & & $n=200$ & $n=300$ & $n=500$ & $n=900$ \\
\hline & $M 1$ & 0.032 & 0.037 & 0.041 & 0.045 \\
\hline & $M 2$ & 0.025 & 0.031 & 0.036 & 0.041 \\
\hline & M3 & 0.016 & 0.024 & 0.026 & 0.033 \\
\hline \multirow{3}{*}{$m=n^{0.9}$} & $M 1$ & 0.031 & 0.035 & 0.040 & 0.045 \\
\hline & $M 2$ & 0.024 & 0.031 & 0.035 & 0.040 \\
\hline & M3 & 0.016 & 0.022 & 0.026 & 0.032 \\
\hline \multicolumn{6}{|c|}{ AR(1) residuals with $\rho=0.2$} \\
\hline \multirow{4}{*}{$m=n^{0.8}$} & & $n=200$ & $n=300$ & $n=500$ & $n=900$ \\
\hline & $M 1$ & 0.045 & 0.046 & 0.049 & 0.053 \\
\hline & $M 2$ & 0.038 & 0.043 & 0.045 & 0.048 \\
\hline & M3 & 0.027 & 0.036 & 0.042 & 0.044 \\
\hline \multirow{3}{*}{$m=n^{0.9}$} & $M 1$ & 0.044 & 0.045 & 0.048 & 0.052 \\
\hline & $M 2$ & 0.036 & 0.042 & 0.044 & 0.047 \\
\hline & M3 & 0.025 & 0.035 & 0.040 & 0.042 \\
\hline \multicolumn{6}{|c|}{$\mathrm{AR}(1)$ residuals with $\rho=0.8$} \\
\hline \multirow{5}{*}{$m=n^{0.8}$} & & $n=200$ & $n=300$ & $n=500$ & $n=900$ \\
\hline & $M 1$ & 0.255 & 0.232 & 0.206 & 0.195 \\
\hline & $M 2$ & 0.158 & 0.141 & 0.129 & 0.121 \\
\hline & M3 & 0.056 & 0.056 & 0.055 & 0.054 \\
\hline & $M 1$ & 0.235 & 0.215 & 0.194 & 0.188 \\
\hline \multirow[t]{2}{*}{$m=n^{0.9}$} & $M 2$ & 0.152 & 0.145 & 0.128 & 0.119 \\
\hline & M3 & 0.055 & 0.057 & 0.059 & 0.057 \\
\hline
\end{tabular}

This table shows the empirical size of the test. $n$ is the simulation sample size and $m$ is the subsample used in the test. $M$ is the bandwidth parameter. $\rho$ is the coefficient in the AR(1) process.

Only when the sample size is relatively small (e.g., $n=200$ in our Monte Carlo experiment), the unit root based tests have reasonably low rates of rejecting the hypothesis of a bubble. But even in this case, if the probability of collapse is high (say, $1-\pi=0.1$ ), the unit root tests still reject the hypothesis of a bubble frequently. As the sample size increases, it is clear from the table that when a bubble pro- cess has the collapsing property, the conventional unit root tests mistakenly reject the null of a bubble in favor of the false alternative of no bubble. The rejection rate is increasing with the sample sizes, indicating asymptotic invalidity of these tests.

On the contrary, as shown in Table 2, the proposed test has much better performance in dealing with a collapsible bubble. As the probability of collapse increases, the power of the proposed test falls only slightly. However, the conventional tests, the ADF and PP tests, have sharply increasing probabilities of falsely rejecting their null of a bubble as the probability of a collapse increases. In addition, the rejection rate of the proposed test increases as the sample size increases, corroborating the asymptotic theory. Both choices of subsamples provide qualitatively similar results.

\section{EMPIRICAL APPLICATIONS}

In this section, we apply the above testing procedure to stock index data from the U.S. and Hong Kong. The sample period covers some of the most conspicuous episodes of stock market crashes.

\subsection{Data description}

We collected weekly price and dividend yield data for the Standard and Poor (S\&P) 500 Index and the Hang Seng Index (Hong Kong) from January 4, 1974 to September 18, 1998 (Friday close to Friday close). The source of the data is the on-line service of Datastream. These markets experienced both crashes as well as relative stability over the sample period. We use the 1-year T-bill yield as the risk free rate for the U.S. market and the Hong Kong market. The yield spread is used as an instrument for the time varying risk premium.

If we define stock market crash as a drop in a major stock index of $10 \%$ or more during a single day, then the

Table 2. Empirical rate of rejecting the null of no bubble (the $\underline{R}$ test)

\begin{tabular}{ccccccccccc}
\hline Prob. of Collapse & 0.02 & 0.04 & 0.05 & 0.07 & 0.1 & 0.02 & 0.04 & 0.05 & 0.07 & 0.1 \\
\hline \multicolumn{7}{c}{$n=200$} & & & & $n=300$ \\
$m=n^{0.8}, M 1$ & 0.79 & 0.78 & 0.77 & 0.74 & 0.72 & 0.89 & 0.87 & 0.87 & 0.865 & 0.855 \\
$m=n^{0.8}, M 2$ & 0.77 & 0.76 & 0.75 & 0.73 & 0.69 & 0.875 & 0.86 & 0.84 & 0.86 & 0.82 \\
$m=n^{0.8}, M 3$ & 0.73 & 0.70 & 0.72 & 0.70 & 0.67 & 0.84 & 0.83 & 0.835 & 0.825 & 0.79 \\
$m=n^{0.9}, M 1$ & 0.79 & 0.72 & 0.70 & 0.76 & 0.77 & 0.87 & 0.88 & 0.85 & 0.82 & 0.77 \\
$m=n^{0.9}, M 2$ & 0.75 & 0.73 & 0.68 & 0.71 & 0.70 & 0.86 & 0.82 & 0.815 & 0.79 & 0.76 \\
$m=n^{0.9}, M 3$ & 0.74 & 0.70 & 0.64 & 0.68 & 0.73 & 0.82 & 0.78 & 0.80 & 0.78 & 0.75 \\
$m=n^{0.8}, M 1$ & 0.88 & 0.86 & 0.87 & 0.85 & 0.865 & 0.95 & 0.95 & 0.92 & 0.915 & 0.90 \\
$m=n^{0.8}, M 2$ & 0.86 & 0.88 & 0.86 & 0.85 & 0.85 & 0.91 & 0.92 & 0.89 & 0.905 & 0.88 \\
$m=n^{0.8}, M 3$ & 0.86 & 0.87 & 0.87 & 0.85 & 0.85 & 0.88 & 0.87 & 0.87 & 0.88 & 0.87 \\
$m=n^{0.9}, M 1$ & 0.87 & 0.875 & 0.87 & 0.84 & 0.825 & 0.92 & 0.91 & 0.91 & 0.89 & 0.90 \\
$m=n^{0.9}, M 2$ & 0.86 & 0.875 & 0.86 & 0.855 & 0.815 & 0.86 & 0.85 & 0.85 & 0.81 & 0.80 \\
$m=n^{0.9}, M 3$ & 0.86 & 0.87 & 0.85 & 0.84 & 0.81 & 0.85 & 0.84 & 0.84 & 0.81 & 0.79 \\
\hline
\end{tabular}

This table shows the empirical rate of rejecting the null of no bubble. $n$ is the simulation sample size and $m$ is the subsample used in the test. $M$ is the bandwidth parameter. 
Table 3. Empirical rate of rejecting the null of a bubble ( $A D F$ and $P P$ )

\begin{tabular}{ccccccccccc}
\hline Prob. of Collapse & 0.02 & 0.04 & 0.05 & 0.07 & 0.1 & 0.02 & 0.04 & 0.05 & 0.07 & 0.1 \\
\hline ADF test & 0.39 & 0.59 & 0.65 & 0.805 & 0.91 & 0.795 & 0.89 & 0.895 & 0.91 & 0.935 \\
PP $t$ test & 0.73 & 0.79 & 0.85 & 0.89 & 0.945 & 0.835 & 0.91 & 0.919 & 0.945 & 0.945 \\
& \multicolumn{7}{c}{$n=500$} & & \multicolumn{7}{c}{$n=900$} \\
ADF test & 0.865 & 0.93 & 0.945 & 0.955 & 0.97 & 0.935 & 0.975 & 0.985 & 0.995 & 0.995 \\
PP $t$ test & 0.875 & 0.93 & 0.955 & 0.97 & 0.975 & 0.94 & 0.975 & 0.985 & 0.995 & 0.995 \\
\hline
\end{tabular}

This table reports the empirical rates of rejecting the null hypothesis of a bubble based on the Augmented Dickey-Fuller test and the Phillips-Perron test. $n$ is the simulation sample size.

U.S. stock market has experienced only two crashes in the 20th Century, one in October 1929 and the other in October 1987. Over our sample period, the S\&P 500 Index, which consists of 500 large capitalization stocks on the New York Stock Exchange, increased 10-fold from 100 to 1,020, with an average annual return of $9.5 \%$. From August 1995 to September 1998, the index produced a $20 \%$ annual rate of return. Expectations on future returns were high at the end of our sample period. However, there were signs that this high level of return might not be sustainable.

The Hong Kong stock market had more than its share of ups and downs. The market crash in 1973 prompted the government to unify the territory's four exchanges to form the Stock Exchange of Hong Kong (SEHK). It is now among the leading stock exchanges in the world and, in terms of market capitalization, the second largest in Asia after the Tokyo Stock Exchange. Over our sample period, the Hang Seng Index increased 17 -fold from 425 to 7,446 , with an average annual return of $11.7 \%$. It experienced crashes in October 1987, June 1989, November 1994 and October 1997. The last crash was preceded by a climactic upward surge on the stock and property markets ahead of the transfer of sovereignty from Britain to China. It was associated with the Asian financial market crises that affected the entire Asia Pacific region. During this dramatic market downturn, the Hang Seng Index dropped from its peak of about 16,700 in August 1997 to about 7,400 in September 1998, a whopping $55 \%$ decline.

\subsection{Testing procedures}

To examine various specifications in modeling market fundamentals, we apply the following two tests to the data sets. The prices and dividends are in logarithms. The upper tail critical values for these tests are calculated based on simulations using a sample of size 2,000 and 20,000 replications.

Test 1: The first test is based on model (21). We assume that market fundamental is not only affected by the dividend series $d_{t}$, but also by the short term interest rate $r_{t}$. We consider the fully modified residuals from the following regressions:

$$
p_{t}=\widehat{\delta}+\widehat{\beta}_{1} d_{t}+\widehat{\beta}_{2} z_{t}+\widehat{u}_{t},
$$

where $z_{t}$ is the inverse interest rate. In this case, the limiting null distributions of the corresponding tests are given by (16) with $S(r)^{\prime}=\left(1, W_{2}(r)\right)$, where $W_{2}(r)$ is a 2 dimensional standard Brownian motion and independent of $W_{1}(r)$ in (16). The corresponding $1 \%$ and $5 \%$ level critical values for this test are 1.285 and 1.041 . The test will reject the null when the calculated test statistic is larger than the corresponding critical value.

Test 2: The second test augments the model in Test 1 by adding an additional variable $\left(A A A_{t}-B B B_{t}\right)$ to capture the time-varying risk-premium effect and consider the following cointegrating regression

$$
p_{t}=\widehat{\alpha}+\widehat{\beta} d_{t}+\widehat{\lambda} z_{1 t}+\widehat{\delta} z_{2 t}+\widehat{u}_{t},
$$

where $z_{1 t}$ is the inverse of the short term interest rate and $z_{2 t}=A A A_{t}-B B B_{t}$.

The test statistic can be constructed in the same way as the previous tests according to formula (15). Under the null hypothesis of no bubble, as $n \rightarrow \infty$, the corresponding test statistic has a limiting distribution similar to (16), but with $W_{2}(r)$ being a 3-dimensional standard Brownian motion independent with $W_{1}(r)$. Asymptotic $1 \%$ and $5 \%$ level critical values are 1.146 and 0.941 respectively.

\subsection{Stationarity of stock prices and dividends}

To detect the existence of bubbles, we first test the (non)stationarity properties of the stock price and the dividend. According to the model described in Section 2, the dividend process and the stock price process are all nonstationary. If there is no bubble in the stock price, the residuals from the cointegrating regression should be stationary $(\mathrm{I}(0))$. If, however, the stock price contains a bubble, then the residuals are not stationary $(\mathrm{I}(0))$, although it is not a traditional unit-root process because the bubble collapses.

A statistic similar to (15) can be used to test for stationarity (or trend stationarity) in the stock and dividend series by simply substituting $\widetilde{u}_{t}^{+}$by the detrended stock prices and the dividends in formula (15) and by replacing $\widehat{\omega}_{u . d}$ by the corresponding long-run variance estimates 
for these series. See the Appendix for a description of this test. We test the stationarity properties of our data using this procedure, and the results are reported in Table 5. (Alternative procedures for testing stationarity were also considered and qualitatively similar results have been obtained.) Since the test is semiparametric and involves a bandwidth choice, we calculate the test statistics for a number of representative values of the bandwidth parameter $M$. Specifically, we calculate the statistics for bandwidth choices $M 1=\left[4(n / 100)^{1 / 4}\right], M 2=\left[6(n / 100)^{1 / 4}\right]$, $M 3=\left[8(n / 100)^{1 / 4}\right]$, and $M 4=\left[12(n / 100)^{1 / 4}\right]$. The tests reject stationarity for statistics larger than a critical value.

We first look at these tests applied to stock prices. The evidence of nonstationarity in the stock prices is very strong. Similar results can also be found using the dividends data. All the calculated statistics (for all bandwidth choices) are much larger than the $5 \%$ level critical values, and thus stationarity should be rejected. Next, we test the stationarity of the first differences of the dividends. It is found that the statistics for all bandwidth choices are less than the $5 \%$ critical values, indicating stationarity. These results support the assumptions of the model and are consistent with the existing empirical results on the nonstationarity of stock prices and dividends. (For more details, see Table 5 in an earlier version of this paper.)

\subsection{Empirical test for bubbles}

We now test the existence of bubbles in stock prices using both Test 1 and Test 2 described in Section 6.2. Since the bandwidth $M$ is an important parameter in the construction of the tests, we examined the tests based on a range of different choices of bandwidth values: $M 1=$ $\left[4(m / 100)^{1 / 4}\right], M 2=\left[6(m / 100)^{1 / 4}\right], M 3=\left[8(m / 100)^{1 / 4}\right]$, $M 4=\left[12(m / 100)^{1 / 4}\right]$, and $M 5=\left[14(m / 100)^{1 / 4}\right]$, where $m$ is the subsample size which is chosen as $m=n^{0.9}$ in our empirical analysis. These lag truncation parameters were chosen because the variance estimates of each time series become reasonably stable by the time we reach $M 5$.

The empirical results are reported in Table 4. In particular, Panel A of Table 4 reports the calculated test statistics for the Hong Kong Hang Seng Index weekly data. Note that the test statistics decline monotonically as $M$ increases. Considering the fact that there is substantial serial correlation in the stock price time series, we are particularly interested in the results when $M$ is large. In the first test (i.e. Test 1), the null hypothesis that there is no bubble is rejected at the $5 \%$ level for all bandwidth choices. Even the minimum statistics (when $M=M 5$ ) is larger than the $5 \%$ critical values. In fact, we reject the null hypothesis even at $1 \%$ level of significance in many cases with Test 1 . When we add the time-varying risk premium covariate, the values of the calculated statistics of Test 2 decrease, when we choose $M=M 5$, the null hypothesis can not be rejected at the $5 \%$
Table 4. Bubble tests for stock prices

\begin{tabular}{llllll}
\hline \multicolumn{7}{c}{ Hong Kong Data } \\
\hline \multirow{7}{*}{ Test 1 } & M1 & M2 & M3 & M4 & M5 \\
p-values & 0.0081 & 1.7549 & 1.5461 & 1.3606 & 1.2266 \\
& The level of $k$ leading to the statistic: & $k^{*}=451$ \\
Test 2 & 1.6943 & 1.3806 & 1.1247 & 1.0075 & 0.9366 \\
p-values & $0.2 \%$ & $1 \%$ & $1.5 \%$ & $2.4 \%$ & $5.1 \%$ \\
& The level of $k$ leading to the statistic: & $k^{*}=447$ \\
\hline \multicolumn{7}{c}{ US Data } \\
\hline Test 1 & 1.8153 & 1.5026 & 1.2522 & 1.1448 & 0.9996 \\
p-value & $0.07 \%$ & $0.6 \%$ & $1.5 \%$ & $2.5 \%$ & $5.2 \%$ \\
Test 2 & The level of $k$ leading to the statistic: & $k^{*}=993$ \\
p-value & 1.3303 & 1.0954 & 0.9029 & 0.8244 & 0.7823 \\
& $0.5 \%$ & $2 \%$ & $5.2 \%$ & $8 \%$ & $14 \%$ \\
& The level of $k$ leading to the statistic: & $k^{*}=924$ \\
\hline
\end{tabular}

This table reports the test results on bubbles in the Hong Kong and U.S. markets. The sample size is 1290 . M is the bandwidth parameter.

level of significance. However, for all other cases, the null hypothesis is rejected at $5 \%$ level. In summary, the tests rejects the null of no bubble for almost all (but one) cases, indicating the possibility of bubbles in the Hong Kong stock market.

Panel B of Table 4 reports the calculated test statistics for the US weekly data. The results of US market turns out to be somewhat different than the Hong Kong market. Again the test statistics decline monotonically as $M$ increases. As shown in these tables, most test statistics are smaller than those for Hong Kong. When we apply Test 1 to the US data, 4 out of 5 statistics are larger than the $5 \%$ critical value. The only case not rejecting bubble at $5 \%$ level is the one using $M 5$. But when we go to $1 \%$ level, three out of the five cases could not reject the null. Such a result is strengthened when we add the time-varying risk premium covariate in the regression, the results of Test 2 indicate that for cases $M=M 3, M 4$, and $M 5$, the null of no bubble could not be rejected at the $5 \%$ level and only the case $M=M 1$ rejects the null at $1 \%$ level. Giving the fact that there is substantial serial correlation in the data and thus a large bandwidth is needed, the evidence of bubbles in the US market is weak and we can not reject the null of no bubble in the second test.

Our tests delivered different results on the US and Hong Kong market. Based on these tests, we conclude that there is some evidence that there were bubbles in the Hong Kong stock market. As discussed in the data section above, the Hong Kong stock market experienced three crashes over the sample period. Our tests can not reject the hypothesis of no bubbles in the US market for some choices of bandwidth values. 


\section{APPENDIX}

\subsection{The fully modified and the nonparametric estimators}

The FM (fully-modified) estimation and related nonparametric estimates have been studied by Phillips and Hansen (1990) and Phillips (1995). For a detailed analysis of these procedures, the readers are referred to these papers. We provide a brief description of them here for convenience.

Under the null hypothesis of no bubble, the partial sum of the residuals in (13) has the following limit

$$
\begin{aligned}
n^{-1 / 2} \sum_{t=1}^{[n r]} \widehat{u}_{t} \Rightarrow & B_{u}(r)-\left[\int_{0}^{1} \underline{B}_{d}(s) d B_{u}(s)+\Delta_{u d}\right] \\
& \times\left[\int_{0}^{1} \underline{B}_{d}(s)^{2} d s\right]^{-1} \int_{0}^{r} \underline{B}_{d}(s) d s
\end{aligned}
$$

where $B_{u}$ is a Brownian motion, $\underline{B}_{d}$ is a demeaned Brownian motion, and $\Delta_{u d}=E\left(\sum_{i=1}^{\infty} u_{0} d_{i}\right)$ is the one-sided long run covariance between $u_{t}$ and $d_{t}$. If the error term $u_{t}$ were known, the limiting distribution of its partial sum process would be $B_{u}(r)$. The second term on the right-hand side of (23) comes from the estimation error of $\widehat{u}_{t}$. Consequently, the limit of the partial sums of $\widehat{u}_{t}$ is a functional of $B_{u}, \underline{B}_{d}$, and depends on $\Delta_{u d}$. These are nuisance parameters. To get rid of the nuisance parameters, we construct the fully modified residual process as

$$
\widetilde{u}_{t}^{+}=p_{t}^{+}-\widehat{\alpha}^{+}-\widehat{\beta}^{+} d_{t},
$$

where $\left(\widehat{\alpha}^{+}, \widehat{\beta}^{+}\right)$is the fully modified estimator of $(\alpha, \beta)$ defined as

$$
\left[\begin{array}{c}
\widehat{\alpha}^{+} \\
\widehat{\beta}^{+}
\end{array}\right]=\left(\sum_{t} w_{t} w_{t}^{\prime}\right)^{-1}\left(\sum_{t} w_{t} p_{t}^{+}-\left[n \widehat{\Delta}_{d u}^{+} \quad 0\right]\right),
$$

$w_{t}=\left(1, d_{t}\right)^{\prime}, p_{t}^{+}=p_{t}-\Delta d_{t}^{\prime} \widehat{\Omega}_{d d}^{-1} \widehat{\Omega}_{d u}, \widehat{\Delta}_{d u}^{+}=\widehat{\Delta}_{d u}-$ $\widehat{\Delta}_{d d} \widehat{\Omega}_{d d}^{-1} \widehat{\Omega}_{d u}$, and $\widehat{\Delta}_{d u}, \widehat{\Delta}_{d d}, \widehat{\Omega}_{d u}$, and $\widehat{\Omega}_{d d}$ are nonparametric kernel estimates (Phillips, 1995) constructed based on a subsample of size $m$ and defined as

$$
\begin{aligned}
& \widehat{\Delta}_{d u}=\sum_{h=0}^{M} k\left(\frac{h}{M}\right) C_{d u}(h), \quad \widehat{\Delta}_{d d}=\sum_{h=0}^{M} k\left(\frac{h}{M}\right) C_{d d}(h), \\
& \widehat{\Omega}_{x u}^{2}=\sum_{h=-M}^{M} k\left(\frac{h}{M}\right) C_{d u}(h), \quad \widehat{\Omega}_{d d}=\sum_{h=-M}^{M} k\left(\frac{h}{M}\right) C_{d d}(h),
\end{aligned}
$$

$k(\cdot)$ is the lag window defined on $[-1,1]$ with $k(0)=1$, $C_{u u}(h)=m^{-1} \sum^{\prime} \widehat{u}_{t} \widehat{u}_{t+h}$ where $\sum^{\prime}$ signifies summation over $1 \leq t, t+h \leq m$, and $M$ is the bandwidth parameter satisfying the property that $M, m \rightarrow \infty$ and $M / m \rightarrow 0$, $n-m \rightarrow \infty$ as the sample size $n \rightarrow \infty$. $C_{d d}, C_{d u}$ are defined in the same way. Candidate kernel functions can be found in standard texts (e.g. Hannan, 1970; Brillinger, 1980; and Priestley, 1981). Similarly, we can construct the nonparametric long-run variance parameter $\widehat{\omega}_{u . d}^{2}$ as

$$
\widehat{\omega}_{u . d}^{2}=\widehat{\omega}_{u}^{2}-\widehat{\Omega}_{u d} \widehat{\Omega}_{d d}^{-1} \widehat{\Omega}_{d u},
$$

where $\widehat{\omega}_{u}^{2}=\sum_{h=-M}^{M} k\left(\frac{h}{M}\right) C_{u u}(h)$. The bubble test can then be constructed based on $\widetilde{u}_{t}^{+}$,

$$
\underline{R}=\max _{k=1, \ldots, n} \frac{k}{\widehat{\omega}_{u . d} \sqrt{n}}\left|\frac{1}{k} \sum_{t=1}^{k} \widetilde{u}_{t}^{+}-\frac{1}{n} \sum_{t=1}^{n} \widetilde{u}_{t}^{+}\right| .
$$

It can be shown that under the null, $\underline{R} \Rightarrow \sup _{0 \leq r \leq 1}|\widetilde{V}(r)|$, where $\tilde{V}(r)=\underline{W}_{d}(r)-r \underline{W}_{d}(1), \underline{W}_{d}(r)=W_{1}(r)-$ $\left[\int_{0}^{1} d W_{1} S^{\prime}\right]\left[\int_{0}^{1} S S^{\prime}\right]^{-1} \int_{0}^{r} S, S(r)^{\prime}=\left(1, W_{2}(r)\right)$, and $W_{1}(r)$ and $W_{2}(r)$ are standard Brownian motions that are independent of each other.

\subsection{Asymptotic behavior of the test under the alternative}

Under the alternative hypothesis, there is a bubble in the stock prices and thus, as shown below, no cointegration between $d_{t}$ and $P_{t}$. Notice that $b_{t}=a_{t} b_{t-1}+u_{t}$, thus, by substitution we have

$$
b_{t}=\varphi_{t, 0} b_{0}+\sum_{l=1}^{t} \varphi_{t, l} u_{l}
$$

where

$$
\varphi_{t, l}=\prod_{j=l+1}^{t} a_{j} .
$$

Assuming that the initial value of bubble $b_{0}=0, b_{t}=$ $\sum_{l=1}^{t} \varphi_{t, l} u_{l}$.

In the presence of a bubble, the partial sum of $\widetilde{u}_{t}^{+}$has different order of magnitude than before. In particular, it can be shown that $\max _{k=1, \ldots, n} \frac{k}{\sqrt{n}}\left|\frac{1}{k} \sum_{t=1}^{k} \widetilde{u}_{t}^{+}-\frac{1}{n} \sum_{t=1}^{n} \widetilde{u}_{t}^{+}\right| \mathrm{di}-$ verge as $n \rightarrow \infty$. However, under $H_{1}$, the nonparametric estimators $\widehat{\omega}_{u}^{2}, \widehat{\Delta}_{x u}$, and $\widehat{\Omega}_{x u}$, diverge as well. In order to preserve the consistency, we need to show that the denominator diverges at a slower rate.

To verify the order of magnitude of the numerator, it suffices to look at 


$$
\begin{aligned}
& \frac{1}{\sqrt{n}} \sum_{t=1}^{[n r]} \widetilde{u}_{t}^{+}= \frac{1}{\sqrt{n}} \sum_{t=1}^{[n r]}\left[p_{t}-\Delta d_{t}^{\prime} \widehat{\Omega}_{d d}^{-1} \widehat{\Omega}_{d u}-\widehat{\alpha}^{+}-\widehat{\beta}^{+} d_{t}\right] \\
&= \frac{1}{\sqrt{n}} \sum_{t=1}^{[n r]}\left[b_{t}-\Delta d_{t}^{\prime} \widehat{\Omega}_{d d}^{-1} \widehat{\Omega}_{d u}-\left(\widehat{\alpha}^{+}-\alpha\right)\right. \\
&\left.-\left(\widehat{\beta}^{+}-\beta\right) d_{t}\right] \\
&= \frac{1}{\sqrt{n}} \sum_{t=1}^{[n r]} b_{t}-\frac{1}{\sqrt{n}} \sum_{t=1}^{[n r]}\left[\Delta d_{t}^{\prime} \widehat{\Omega}_{d d}^{-1} \widehat{\Omega}_{d u}+\left(\widehat{\alpha}^{+}-\alpha\right)\right. \\
&\left.+\left(\widehat{\beta}^{+}-\beta\right) d_{t}\right] .
\end{aligned}
$$

By straightforward calculations, we can verify the order of magnitude of each component. For example, for the first term,

$$
\frac{1}{\sqrt{n}} \sum_{t=1}^{[n r]} b_{t}
$$

we calculate its second moment. In particular,

$$
E\left[\frac{1}{\sqrt{n}} \sum_{t=1}^{[n r]} b_{t}\right]^{2}=\frac{1}{n} \sum_{t=1}^{[n r]} E b_{t}^{2}+\frac{2}{n} \sum_{t=1}^{[n r]} \sum_{s=t+1}^{[n r]} E b_{t} b_{s}
$$

by a direct calculation of expectations we have

$$
E b_{t}^{2}=\sigma_{u}^{2}\left(\Delta^{t}-1\right) /(\Delta-1),
$$

and for $s>t$

$$
E b_{t} b_{s}=\sigma_{u}^{2}\left(\Delta^{t}-1\right) /\left[(\Delta-1) \delta^{s-t}\right]
$$

where

$$
\Delta=E\left(a_{t}^{2}\right)=\operatorname{Var}\left(a_{t}^{2}\right)+1 / \delta^{2}=\sigma_{a}^{2}+1 / \delta^{2}>1 .
$$

Thus

$$
E\left[\frac{1}{\sqrt{n}} \sum_{t=1}^{[n r]} b_{t}\right]^{2}=O\left(\frac{\Delta^{[n r]}}{n}\right)
$$

and $(24)$ is of order $O_{p}\left(\sqrt{\frac{\Delta^{[n r]}}{n}}\right)$. We can verify that the order of magnitude of

$$
\frac{1}{\sqrt{n}} \sum_{t=1}^{[n r]}\left[\Delta d_{t}^{\prime} \widehat{\Omega}_{d d}^{-1} \widehat{\Omega}_{d u}+\left(\widehat{\alpha}^{+}-\alpha\right)+\left(\widehat{\beta}^{+}-\beta\right) d_{t}\right]
$$

is also $O_{p}\left(\sqrt{\frac{\Delta^{[n r]}}{n}}\right)$.

For the nonparametric estimator, we consider

$$
\widehat{\omega}_{u}^{2}=\sum_{h=-M}^{M} k\left(\frac{h}{M}\right) C_{u u}(h),
$$

where

$$
C_{u u}(h)=C_{u u}(h)=m^{-1} \sum_{1 \leq t, t+h \leq m} \widehat{u}_{t} \widehat{u}_{t+h} .
$$

Again, by direct calculations, it can be verified that $m^{-1} \sum_{1 \leq t, t+h \leq m} \widehat{u}_{t} \widehat{u}_{t+h}=O_{p}\left(\frac{\Delta^{m}}{m}\right)$ and $\widehat{\omega}_{u}^{2}=O_{p}\left(M \Delta^{m} / m\right)$. Other nonparametric estimates can be verified similarly (also see Xiao and Phillips (1998) for related discussions on periodogram averages of integrated processes). Consequently, the testing statistic is of order $O_{p}\left(\Delta^{(n-m) / 2} / \sqrt{M}\right)$, which diverges to $\infty$ under our assumptions.

\subsection{Stationarity test}

The testing procedure can also be used to detect stationarity. Suppose that we want to test the hypothesis that a time series $z_{t}$ is stationary around a deterministic trend $x_{t}$. We detrend $z_{t}$ by least squares regression

$$
z_{t}=\widehat{\gamma}^{\prime} x_{t}+\widehat{y}_{t}
$$

and denote the detrended time series as $\widehat{y}_{t}=z_{t}-\widehat{\gamma}^{\prime} x_{t}$, where $\widehat{\gamma}=\left[\sum_{t} x_{t} x_{t}^{\prime}\right]^{-1}\left[\sum_{t} x_{t} z_{t}\right]$. The following statistic can then be used in testing (trend) stationarity in time series

$$
\underline{S}_{n}=\max _{k=1, \ldots, n} \frac{k}{\widehat{\omega}_{y} \sqrt{n}}\left|\frac{1}{k} \sum_{t=1}^{k} \widehat{y}_{t}-\frac{1}{n} \sum_{t=1}^{n} \widehat{y}_{t}\right| .
$$

Under stationarity, as $n \rightarrow \infty$,

$$
\underline{S}_{n} \Rightarrow \sup _{0 \leq r \leq 1}\left|\widetilde{W}_{X}(r)\right|
$$

where $\widetilde{W}_{X}(r)=W(r)-\left[\int_{0}^{1} d W(s) X(s)^{\prime}\right]\left[\int_{0}^{1} X(s) X(s)^{\prime} d s\right]^{-1} \times$ $\int_{0}^{r} X(s) d s$.

The critical values of $\underline{S}_{n}$ for the leading case of a linear trend are calculated by a direct simulation using a sample size of 3,000 and 50,000 replications and the 10\%,5\%, and $1 \%$ level upper tail critical values are $0.827,0.901,1.041$, respectively.

\section{Received 29 February 2008}

\section{REFERENCES}

BARsky, R. and Delong, B. (1993). Why does the stock market fluctuate? Quarterly Journal of Economics 108 291-311.

Bekaert, G. and Wu, G. (2000). Asymmetric volatility and risk in equity markets. Review of Financial Studies 13 1-42.

Blanchard, O. J. and Watson, M. W. (1982). Bubbles, rational expectations, and financial markets. Crises in the Economic and $\mathrm{Fi}$ nancial Structure, Wachtel, P. (Ed.), Lexington, Mass.: Lexington Books.

Brillinger, D. R. (1980). Time Series: Data Analysis and Theory, New York: Holt, Rinehart and Winston. MR0443257

CAmerer, C. (1989). Bubbles and fads in asset prices. Journal of Economic Surveys 3 3-41. 
Campbell, J. Y. and Cochrane, J. H. (1999). By force of habit: A consumption-based explanation of aggregate stock market behavior. Journal of Political Economy 107 205-251.

Campbell, J. Y., Lo, A. W., and MacKinlay, A. C. (1997). The Econometrics of Financial Markets, Princeton, NJ: Princeton University Press.

Campbell, J. Y. and Shiller, R. J. (1987). Cointegration and tests of present value models. Journal of Political Economy 95(5) 1062-88.

Campbell, J. Y. and Shiller, R. J. (1988). The dividend-price ratio and expectations of future dividends and discount factors. Review of Financial Studies 1 195-228.

Cochrane, J. H. (1991). Volatility tests and efficient markets. Journal of Monetary Economics 27 463-485.

Cox, J. C. and Ross, S. A. (1976). The valuation of options for alternative stochastic processes. Journal of Financial Economics $\mathbf{3}$ $145-166$.

Dezhbakhsh, D. and Demirguckunt, J. (1990). On the presence of speculative bubbles in stock prices. Journal of Financial and Quantitative Analysis (March) 101-112.

Diba, B. and Grossman, H. (1988). Explosive rational bubbles in stock prices. American Economic Review 78 520-530.

Dickey, D. A. and Fuller, W. A. (1979). Distribution of estimators for autoregressive time series with a unit root. Journal of the American Statistical Association 74 427-431. MR0548036

Donaldson, R. and Kamstra, M. (1996). A new dividend forecasting procedure that rejects bubbles in asset prices: The case of 1929's stock crash. Review of Financial Studies 9 333-383.

Engle, R. and Granger, C. (1987). Cointegration and error correction: Representation, estimation and testing. Econometrica $55251-$ 276. MR0882095

Evans, G. (1986). A test for speculative bubbles in the sterling dollar exchange rate: 1981-1984. American Economic Review 76 222-230.

Evans, G. (1991). Pitfalls in testing for explosive bubbles in asset prices. American Economic Review 81(4) 922-930.

FloOd, R. P. and Garber, P. M. (1980). Market fundamentals versus price-level bubbles: The first tests. Journal of Political Economy $\mathbf{8 8}$ $745-770$

Flood, R. P. and Hodrick, R. J. (1986). Asset price volatility, bubbles, and process switching. Journal of Finance 41 831-842.

Froot, K. A. and Obstfeld, M. (1991). Intrinsic bubbles: The case of stock prices. American Economic Review 81 1189-1214.

Gordon, M. (1962). The Investment, Financing and Valuation of Corporations, Homewood, Ill.: Irwin.

Hannan, E. (1970). Multiple Time Series, New York: Wiley. MR0279952

KIM, K. and SCHMidT, P. (1993). Unit root tests with conditional heteroskedasticity. Journal of Econometrics 59 287-300.

Kwiatkowski, D., Phillips, P. C. B., Schmidt, P., and Shin, Y. (1992). Testing the null hypothesis of stationarity against the alternative of a unit root: How sure are we that economic time series have a unit root? Journal of Econometrics 54 159-178.

McQueen, G. and Thorley, S. (1994). Bubbles, stock returns, and duration dependence. Journal of Financial and Quantitative Analysis 29 186-197.

Nicholls, D. and Quinn, B. (1982). Random Coefficient Autoregressive Models: An introduction, Springer-Verlag. MR0671255

Phillips, P. C. B. (1995). Fully modified least squares and vector autoregression. Econometrica 63 1023-1078. MR1348513

Phillips, P. C. B. and Hansen, B. (1990). Statistical inference in instrumental variables regressions with $\mathrm{I}(1)$ processes. Review of Eco- nomic Studies 57 99-125. MR1034653

Phillips, P. C. B. and Ouliaris, S. (1991). Asymptotic properties of residual based tests for cointegration. Econometrica 58 165-194. MR1046923

Phillips, P. C. B. and Perron, P. (1988). Testing for unit roots in time series regression. Biometrika 75 335-346. MR0946054

Ploberger, W., Kramer, W., and Koutrus, K. (1989). A new test for structural stability in the linear regression model. Journal of Econometrics 40 307-318. MR0994952

Pourahmadi, M. (1986). On the stationarity of the solution of a doubly stochastic model. Journal of Time Series Analysis 7(2) 123-131. MR0847822

Priestley, M. B. (1981). Spectral Analysis and Time Series, Academic Press.

Rappoport, P. and White, E. (1993). Was there a bubble in the 1929 stock market? Journal of Economic History $\mathbf{5 3}$ 549-574.

SHILler, R. (1981) Do stock prices move too much to be justified by subsequent changes in dividends. American Economic Review (June) 421-436.

SEN, P. K. (1980). Asymptotic theory of some tests for a possible change in regression slope occurring at an unknown time point. Zeitschrift fur Wahrscheinlichkeitstheories und Verwandte Gebiete 52 203-218. MR0568268

SHin, Y. (1994). A residual based test of the null of cointegration against the alternative of no cointegration. Econometric Theory 10 91-115. MR1279692

Tirole, J. (1982). On the possibility of speculation under rational expectations. Econometrica 50 1163-1181.

West, K. (1988). A specification test for speculative bubbles. Quarterly Journal of Economics 102 553-580.

Wu, Y. (1997). Rational bubbles in the stock market: Accounting for the U.S. stock price volatility. Economic Inquiry 35 309-319.

XIAO, Z. (2001) Testing the null hypothesis of stationarity against an autoregressive unit root alternative. Journal of Time Series Analysis 22 87-105. MR1816318

Xiao, Z. and Phillips, P. C. B. (2002). A CUSUM test for cointegration using regression residuals. Journal of Econometrics 108 43-61. MR1894218

\section{Guojun Wu}

Bauer College of Business

University of Houston

Houston, TX 77204

USA

Zhijie Xiao

Department of Economics

Boston College

Chestnut Hill, MA 02467

USA

Phone: (617) 552-1709

E-mail address: xiaoz@bc.edu

and

Tsinghua University

Beijing, China 